\title{
MODELLING ASTRID-LIKE SODIUM-COOLED FAST REACTOR WITH SERPENT-DYN3D CODE SEQUENCE
}

\author{
Wojciech Rydlewicz ${ }^{1}$, Emil Fridman ${ }^{2}$, and Eugene Shwageraus ${ }^{1}$ \\ ${ }^{1}$ University of Cambridge, Department of Engineering, Cambridge Nuclear Energy Centre, \\ Trumpington Street, CB2 1PZ, Cambridge, UK \\ ${ }^{2}$ Helmholtz-Zentrum Dresden-Rossendorf, Reactor Safety Division, Institute of Resource \\ Ecology, Bautzner Landstraße 400, 01328 Dresden, Germany \\ wojciech@rydlewicz.pl, e.fridman@hzdr.de, es607@cam.ac.uk
}

\begin{abstract}
This study explores the feasibility of applying the Serpent-DYN3D sequence to the analysis of Sodium-cooled Fast Reactors (SFRs) with complex core geometries, such as the ASTRIDlike design. The core is characterised by a highly heterogeneous configuration and was likely to challenge the accuracy of the Serpent-DYN3D sequence. It includes axially heterogeneous fuel assemblies, non-uniform fuel assembly heights and large sodium plena. Consequently, the influence of generation and correction methods of various homogenised, few-group crosssections (XS) on the accuracy of the full-core nodal diffusion DYN3D calculations is presented. An attempt to compare the approximate time effort spent on models preparation against the accuracy of the result is made. Results are compared to reference full-core Serpent MC (Monte Carlo) solutions. Initially, XS data was generated in Serpent using traditional methods (2D single assemblies and 2D super-cells). Full core calculations and MC simulations offered a moderate agreement. Therefore, XS generation with 2D fuel-reflector models and 3D single assembly models was verified. Super-homogenisation (SPH) factors for XS correction were applied. In conclusion, the performed work suggests that Serpent-DYN3D sequence could be used for the analysis of highly heterogeneous SFR designs similar to the studied ASTRID-like, with an only small penalty on the accuracy of the core reactivity and radial power distribution prediction. However, the XS generation route would need to include the correction with SPH factors and generation of XS with various MC models, for different core regions. At a certain point, there are diminishing returns to using more complex XS generation methods, as the accuracy of full-core deterministic calculations improves only slightly, while the time effort required increases significantly.
\end{abstract}

KEYWORDS: Group constant generation, Serpent, DYN3D, Monte Carlo, ASTRID

\section{INTRODUCTION}

Some recently conducted studies have shown the potential of the Monte Carlo (MC) Serpent code to generate homogenised, few-group cross-sections (XS) for full core nodal diffusion analyses of conventional designs of Sodium-cooled Fast Reactors (SFRs) using the DYN3D package. They indicate that there is merit in applying Serpent MC code [1-8]. This paper concerns the feasibility of applying the SerpentDYN3D sequence to the calculations of SFR cores with more complex geometries. Therefore, the ASTRIDlike core design is studied. It is characterised by a more complicated arrangement than the SFR cores 
analysed so-far [1-8], for example, Phoenix reactor in France - a deployed SFR [4]. The goal of the ASTRID reactor was to demonstrate safety and superior operability properties of Gen-IV SFR reactors [9]. Experience gained from the operation of experimental prototype and commercial [10-13] size SFRs were used to support the design of the ASTRID reactor. The examined core includes axially heterogeneous fuel assemblies, non-uniform fuel assembly heights and large sodium plena. These features were likely to challenge the accuracy of the analysis with the Serpent-DYN3D sequence. Therefore, it was considered worthwhile to perform a study to determine the influence of various XS generation and correction methods on the results of full-core (rodded and un-rodded) deterministic calculations.

Increasing complexity and the time effort spent on XS generation should result in better accuracy of these calculations. Consequently, this work attempts to compare the approximate time effort spent against the achieved, quantitatively assessed accuracy. Since the simulations and calculations were performed on computer hardware of various specifications, the issues of time effort had to be subject to a qualitative assessment. Different configurations of few-group XS were applied. Varying approaches to the generation of few-group XS based on Serpent MC simulations were used. They included implementing 2D single assemblies (infinite lattice), 2D super-cells, 2D fuel-reflector models and taking advantage of Serpent MC code 3D capabilities, with 3D single assembly models. Simultaneously, the influence of the superhomogenisation (SPH) factors $[3,4]$ correction method on the full core calculations was investigated. SPH factors were implemented to correct the XS generated with 2D super-cells. All of the ASTRID-like full core nodal diffusion solutions in DYN3D were compared against full core Serpent MC simulations.

The structure of the paper is as follows. Section 2 presents a brief core geometry description, followed by methodologies used in the generation and correction of XS. In Section 3 the results of the entire core calculations (radial and axial power distribution) are shown. Section 4 provides a summary and remarks on the study.

\section{METHODOLOGY}

\subsection{ASTRID-like geometry}

The considered ASTRID-like core is a medium-scale (1500 MW of thermal power) pool-type SFR. A heterogeneous core design was created to enhance the safety features of the reactor, by lowering the sodium void coefficient [14]. The core consists of 883 assemblies, the total height of which is $301.53 \mathrm{~cm}$. Axial and radial layouts are presented in Figure 1.

a)

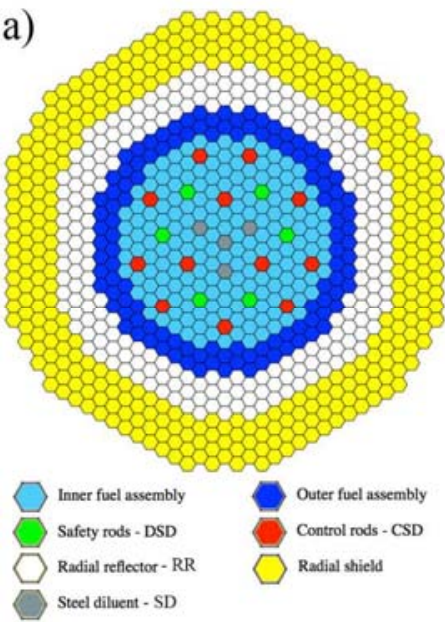

b)

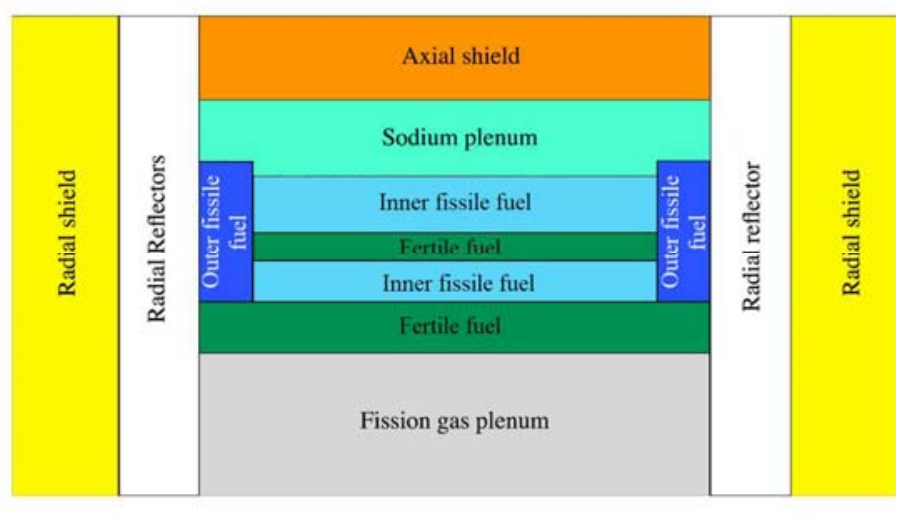

Figure 1. An outline of the a) radial and b) axial layout of the ASTRID-like core [17]. 
The core is divided into regions composed of outer fuel assemblies, inner fuel assemblies, safety rods (DSD), control rods (CSD), radial reflectors (RR), radial shield, steel diluent (SD), axial shield, sodium plena and fission gas plena (Figure 1). In the nominal operating conditions, assumed in the analysis, the temperature of the fissile fuel is $1227^{\circ} \mathrm{C}$, the temperature of the fertile fuel is $627^{\circ} \mathrm{C}$; clad, coolant and wrapper temperatures are $475^{\circ} \mathrm{C}$, while diagrid is at $400{ }^{\circ} \mathrm{C}$. A detailed description of the geometry can be found in the works by Bortot et al. [15], Gabrielli et. al [16] and Rydlewicz [17].

\subsection{Generation of few-group $X S$}

Several approaches were adopted to generate few-group, homogenised cross-sections (XS) for subassemblies or core regions. The few-group XS included macroscopic reactions cross-sections, scattering matrices and diffusion coefficients. Generation methods incorporated taking advantage of Serpent MC 2D and 3D modelling capabilities with:

- 2D single assembly (infinite lattice), for fissile and fertile fuel sub-assemblies (SA). Fuel subassemblies were modelled as a 2D infinite lattice, with reflective boundary condition. Such an approach required using various models for different fuel sub-assemblies' layers. Moreover, in this approach, the spectral effects of the axial non-multiplying regions in the fuel assemblies were not accounted for. An exemplary model of a lattice is shown in Figure $2 a$ and Figure $2 b$.

- 2D super-cell models, for non-multiplying regions and, in one case, fertile fuel SA. Non-multiplying regions included sodium plena in fuel assemblies, sodium plena in control rod followers, control rods, safety rods, axial shield, gas plenum, steel diluent, radial reflectors and radial shield. The cells were modelled as 2D central non-multiplying hexagonal regions surrounded by fuel sub-assemblies, with a reflective boundary condition. Exemplary models are presented in Figure 2b.

- 2D fuel-reflector models, for outermost fuel sub-assemblies and innermost radial reflectors facing them. Previous research [2] showed a non-negligible spectral transition between the outermost fuel SA and radial reflectors. There is a thermalised neutron influx into the outermost fuel sub-assemblies, and consequently, the spectrum is 'softened' (more thermal). Applying only 2D or 3D single assembly models (infinite lattice) to represent the outermost fuel sub-assemblies does not account for this fact. Hence, it was necessary to introduce models accounting for this phenomenon. In a 2D fuel-reflector model a black boundary condition was applied radially (x-direction), while a reflective boundary condition was used in the y-direction (Figure 2c). Group constants (XS) were homogenised over the outermost fuel sub-assemblies and/or innermost radial reflectors.

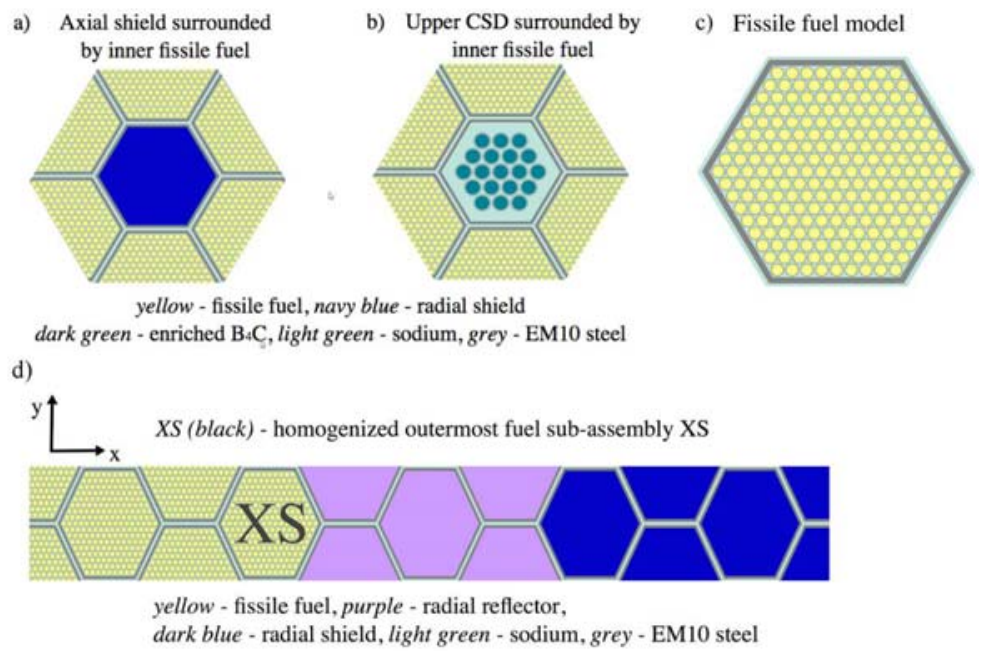

Figure 2. Serpent MC models of a), b) 2D super-cells, c) a 2D single assembly model and d) a 2D fuel-reflector model [17]. 
- 3D single assembly (infinite lattice). Homogenised, few-group cross-sections (XS) for fuel subassemblies (fissile and fertile) were also calculated using 3D single assembly models. Separate models for the inner fuel assemblies and the outer fuel assemblies were created. Reflective radial and black axial boundary conditions were applied. Group constants were homogenised only over the fuel (fissile and fertile) sub-assemblies. The design of these Serpent MC models was dictated by the need to approximate the real (actual in-core) conditions as realistically as possible. Consequently, the spectral effects on the $\mathrm{XS}$ in the fuel sub-assemblies, present due to the proximity (in the axial direction) of the non-multiplying regions (gas plena, sodium plenum and axial shield), are accounted for. Moreover, such models allowed to simultaneously generate XS for all of the fissile and fertile fuel sub-assemblies.

\subsection{Correction of few-group XS with super-homogenisation factors (SPH)}

The process of homogenisation over a region or a sub-assembly may result in the loss of accuracy of flux and reaction rates values in comparison with a heterogeneous solution. Consequently, nodal diffusion solutions of entire 3D cores (mainly characterised by a heterogeneous design and operating in fast neutron spectrum), which base on applying few-group XS, may be burdened with errors. Consequently, the superhomogenisation (SPH) factors method is applied to correct few-group XS of in core regions of particular interest.

SPH was suggested by Kavenoky [18] and later extended by Hebert [19,20]. It has been typically implemented for generation of few-group XS for Light Water Reactors [21,22]. However, it has recently been applied by Nikitin et al. for the analysis of a $3600 \mathrm{MW}$ of thermal power mixed-oxide (MOX) SFR core specified by SFR Benchmark Task Force of OECD/NEA [2]. A precise description of the SPH factors method, applied in this study, is available in Nikitin et al. [2]. It consists of an iterative procedure of multiplication of few-group XS, which were initially obtained from a heterogeneous reference model (in this study created by Serpent) by super-homogenisation factors. These few-group XS are continuously reapplied to a homogeneous model (in DYN3D), which is re-calculated and compared against the heterogeneous model. In the correction process, calculations are repeated until equivalence is obtained between the total reaction rates (in all energy groups and sub-regions) in a heterogeneous reference model and a homogeneous model [18] (Figure 2). In this research, only 2D super-cells were subjected to SPH corrections (Figure 3).

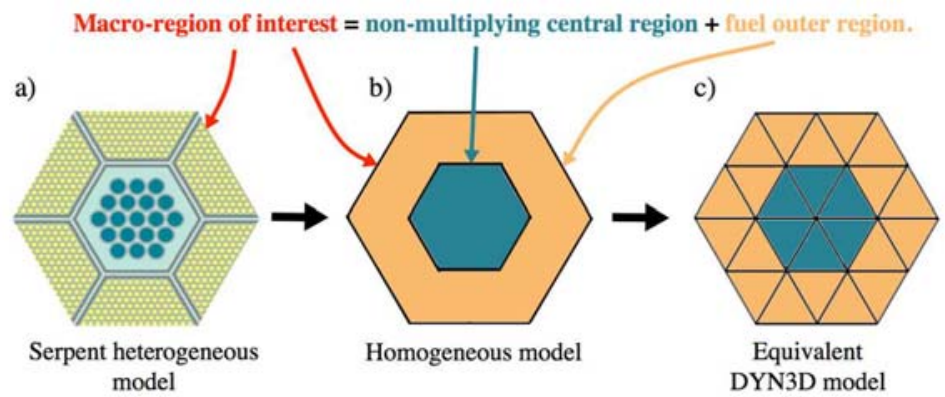

Figure 3. Serpent and DYN3D models of 2D super-cells used to correct the few-group XS [17].

\subsection{Energy group structure}

Based on experience from previous studies [1-3], a 24-group energy structure was adopted. It was created by merging thermal energy groups of the ECCO 33-group energy structure (groups 25 to 33) with group 24 to create a single thermal energy group with the upper boundary of $1.4863 \mathrm{E}-04 \mathrm{MeV}$ [1]. Group 24 was designated as a cut-off as below this energy the statistical uncertainty of the neutron flux increased above $1 \%$. Details can be found in the paper by Fridman and Shwageraus [1]. 


\subsection{Serpent MC reference simulations}

The un-rodded and fully rodded 3D ASTRID-like core simulations in Serpent were completed with the neutron population set to 500000, 1000 active runs and 200 inactive runs. A reference 3D full core solution in an un-rodded state was attained by averaging 15 simulation runs, while in the case of a fully rodded state 1 run was considered. The number of inactive runs was based on the convergence of Shannon entropy investigated during previous research on the Uranium Carbide SFR core by Fridman and Shwageraus [1].

\subsection{DYN3D models' configuration}

Core models in DYN3D were axially divided into 35 layers for an un-rodded core and 37 layers in a fully rodded core. The number of the layers depended on the axial layout of the inner core and outer core fuel sub-assemblies, and the axial position of control rods and safety rods. In each subsequent calculation of a distinct DYN3D ASTRID-like core model, more complex methods of few-group XS generation were applied. In both cases, 19 different models (with specific XS generated or corrected by various means) of both the rodded and the un-rodded core were tested. More complex few-group XS models required further efforts in the preparations stage. However, they were expected to more accurately capture the actual in-core conditions of a particular core zone or a sub-assembly (macroscopic cross-sections, scattering matrices and diffusion coefficients), thus positively influencing the precision of the nodal diffusion solution of the full core. Finally, each DYN3D full core model was compared against a Serpent MC reference simulation.

\section{RESULTS AND DISCUSSION}

\subsection{Results division and qualitative effort assessment}

Among all of the 19 DYN3D models, for the rodded and un-rodded core respectively, there were similar ones, with XS generated at a comparable level of complexity. Therefore, to facilitate the discussion, all of the models are grouped into representative Models \#1 to \#6 (for both un-rodded and rodded cores respectively). In each Model, the results of the best performing approach are shown. Models are ranked by the efforts required to generate an entire set of XS (from \#1, least effort, to \#6, greatest effort) in Table 1.

Table I. XS generation and correction methods for various core regions.

\begin{tabular}{|c|c|c|c|c|c|c|c|}
\hline \multirow{2}{*}{$\begin{array}{c}\text { Model } \\
\#\end{array}$} & \multicolumn{4}{|c|}{ Fuel regions or sub-assemblies } & \multicolumn{3}{|c|}{$\begin{array}{c}\text { Non-multiplying regions or sub- } \\
\text { assemblies }\end{array}$} \\
\hline & $\begin{array}{l}\text { 2D single } \\
\text { assemb. }\end{array}$ & $\begin{array}{l}2 D \text { fuel- } \\
\text { reflector }\end{array}$ & $\begin{array}{l}\text { 3D single } \\
\text { assemb. }\end{array}$ & $\begin{array}{l}\text { 2D super-cells } \\
+\mathrm{SPH}\end{array}$ & $\begin{array}{l}\text { 2D super- } \\
\text { cells }\end{array}$ & $\begin{array}{l}\text { 2D super cells } \\
+ \text { SPH }\end{array}$ & $\begin{array}{l}2 D \text { fuel- } \\
\text { reflector }\end{array}$ \\
\hline 1 & All & - & - & - & All & - & - \\
\hline 2 & $A l l^{e x c l . ~} 1$ & $\begin{array}{l}{ }^{1} \text { Outermost } \\
\text { fertile }\end{array}$ & - & - & All & - & - \\
\hline 3 & - & - & All & - & All & - & - \\
\hline 4 & - & - & All & - & All ${ }^{\text {excl. } 2}$ & $\begin{array}{l}{ }^{2} C S D^{*}, D S D^{*} \\
S D^{*}\end{array}$ & - \\
\hline 5 & - & - & $A l l^{\text {excl. } 3}$ & $\begin{array}{l}{ }^{3} \text { Fuel adjacent } \\
\text { to } S D^{*}\end{array}$ & All ${ }^{\text {excl. } 4}$ & $\begin{array}{l}{ }^{4} C S D^{*}, D S D^{*} \\
S D^{*}\end{array}$ & - \\
\hline 6 & - & $\begin{array}{c}{ }^{5} \text { Outermost } \\
\text { fertile }\end{array}$ & $A l l^{\text {excl. } 56}$ & $\begin{array}{c}{ }^{6} \text { Fuel adjacent } \\
\text { to } C S D^{*}, D S D^{*}, \\
S D^{*}\end{array}$ & $A l l^{\text {excl. } 56}$ & $\begin{array}{c}{ }^{5} C S D^{*}, D S D^{*} \\
S D^{*}\end{array}$ & $\begin{array}{l}{ }^{6} \text { Innermo } \\
\text { st } R R^{*}\end{array}$ \\
\hline
\end{tabular}

"SD - Steel diluent, RR - Radial reflector, CSD - control rods, DSD - safety rods. 
The wider the variety of Serpent models for XS set generation is, and the larger these models are, the longer the calculations to create this set, on the same CPU, take. The same rules apply for SPH factors - more CPU time is needed if they are employed to correct the distinct 2D super-cell generated XS. The proposed ranking is a qualitative division. It is crucial in the context of the assessment of efforts vs. accuracy. This ranking also happens to correspond to the increasing accuracy of the results of the full-core deterministic calculations.

\subsection{Un-rodded and rodded core results accuracy}

The changes in the values of RMS (axial and radial power peaking factors, Serpent vs. DYN3D) and k-eff (DYN3D vs. Serpent) in the function of Model \# are presented in Figure 5.

For the un-rodded core, Model \#1 resulted in an RMS error of radial power peaking factor of $0.93 \%$ (Figure $4 a$ ) and the k-eff difference of equal to $-447 \mathrm{pcm}$ (Figure $4 \mathrm{~b}$ ). The power map is presented in Figure 5a. The best performing approach, Model 6\#, offered the RMS error of power peaking of $0.59 \%$ (Figure 4a) and the reactivity discrepancy of $-119 \mathrm{pcm}$ (Figure $4 \mathrm{~b}$ ). The power map is presented in Figure $5 \mathrm{~b}$.

In the case of the rodded core, Model \#1 the RMS of the radial power map difference was $2.10 \%$ (Figure $5 a$ ), while the k-eff difference was at $-682 \mathrm{pcm}$ (Figure 5b). Model \#6 was the best performing approach, with k-eff at -249 pcm (Figure 5b) and RMS of the radial power map of $0.90 \%$ (Figure 5a).

In the case of the axial power distribution (and its RMS value) of both the un-rodded and rodded core, the gradual enhancements in XS generation showed varying, yet minor, improvements with regards to the reference MC solution (Figure 5c and 5d).

\subsection{Discussion on results improvements}

Introduction of 3D single assembly models improved k-eff significantly (improvement, from Model \#2 to Model 3\#, of $207 \mathrm{pcm}$ and $129 \mathrm{pcm}$, for the un-rodded and rodded cores respectively). Notable results were also attained with the application of SPH-corrected XS for non-multiplying regions of control rods, safety rods and steel diluent (an improvement from Model \#3 to Model \#4, by $76 \mathrm{pcm}$ and $130 \mathrm{pcm}$, for the unrodded and rodded cores respectively). Finally, in the rodded core case, a similar development was noted when SPH-corrected XS were used for fuel around the still diluent (Model \#4 to Model \#5), as the k-eff difference was lower by $115 \mathrm{pcm}$.

In case of the radial power map, initially, 2D fuel-reflector models, as in work by Fridman and Shwageraus [1], were tested to correct the undervaluation of power in the outer core regions (Figure 4a). XS generated this way advanced only the outermost fuel assemblies peaking factors coherence, not k-eff, and only if they were used for fertile fuel regions. Moreover, it was revealed during the study that XS created with 2D fuelreflectors should not be used in connection with 3D single assembly models, unless SPH-corrected XS for non-multiplying regions of control rods (CSD), safety rods (DSD) and steel diluents (SD) are introduced in the XS set. Such a connection improved the k-eff, but worsen the coherence of the power peaking factors in the inner core region. Consequently, 2D fuel-reflector models were not applied in Models \#3 - \#5. The greatest advancement in terms of RMS values of power peaking factors happened with the application of $\mathrm{SPH}$-corrected XS in Model \#4. Further attempts to increase the coherence of the results were also successful, yet there were diminishing returns in increasing the efforts of more distinct XS generation.

In the case of axial power distribution, no specific pattern was recognised (Figure $4 c$ and $4 d$ ). 
a)

Radial power dist. difference

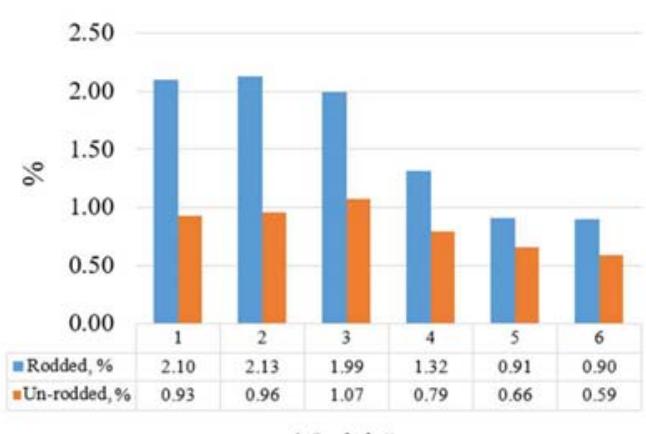

Model \#

c)

3.5

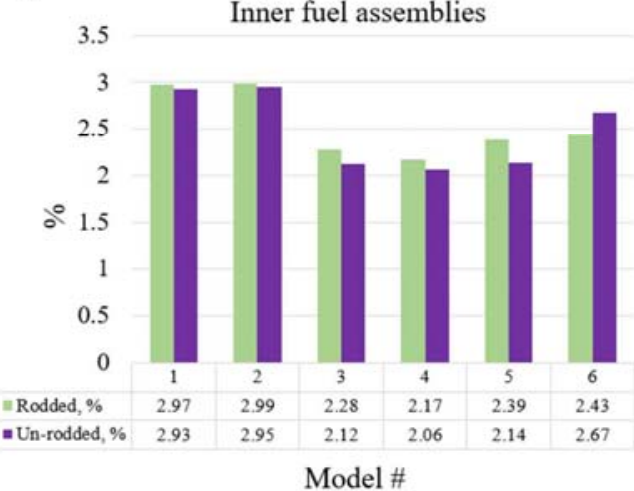

b)

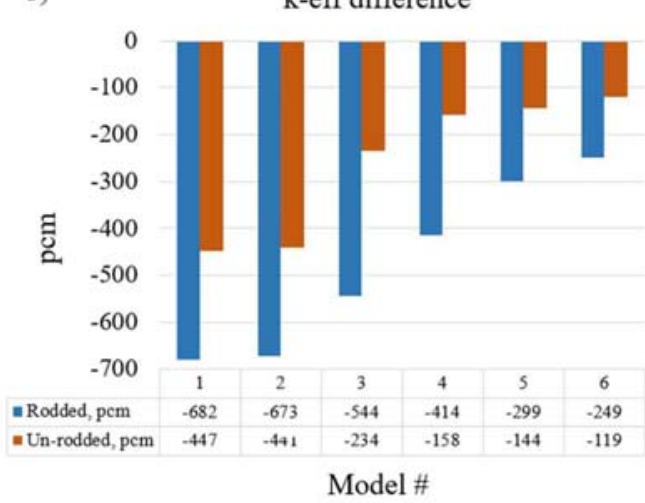

d)

d) Axial power dist. difference

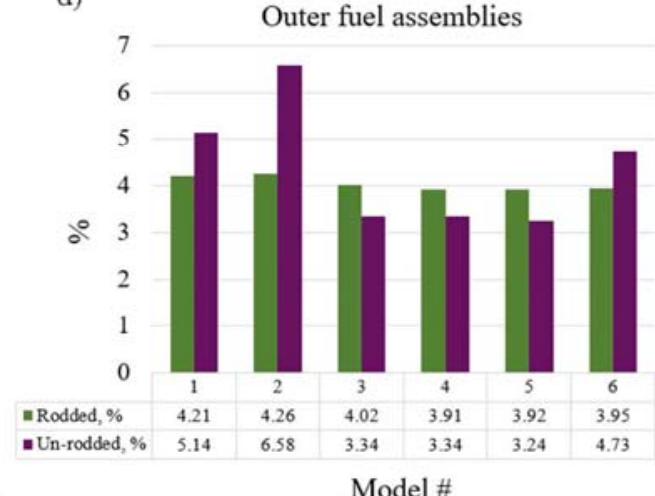

Figure 4. RMS and k-eff differences between the DYN3D nodal diffusion calculations and reference full-core MC simulations.
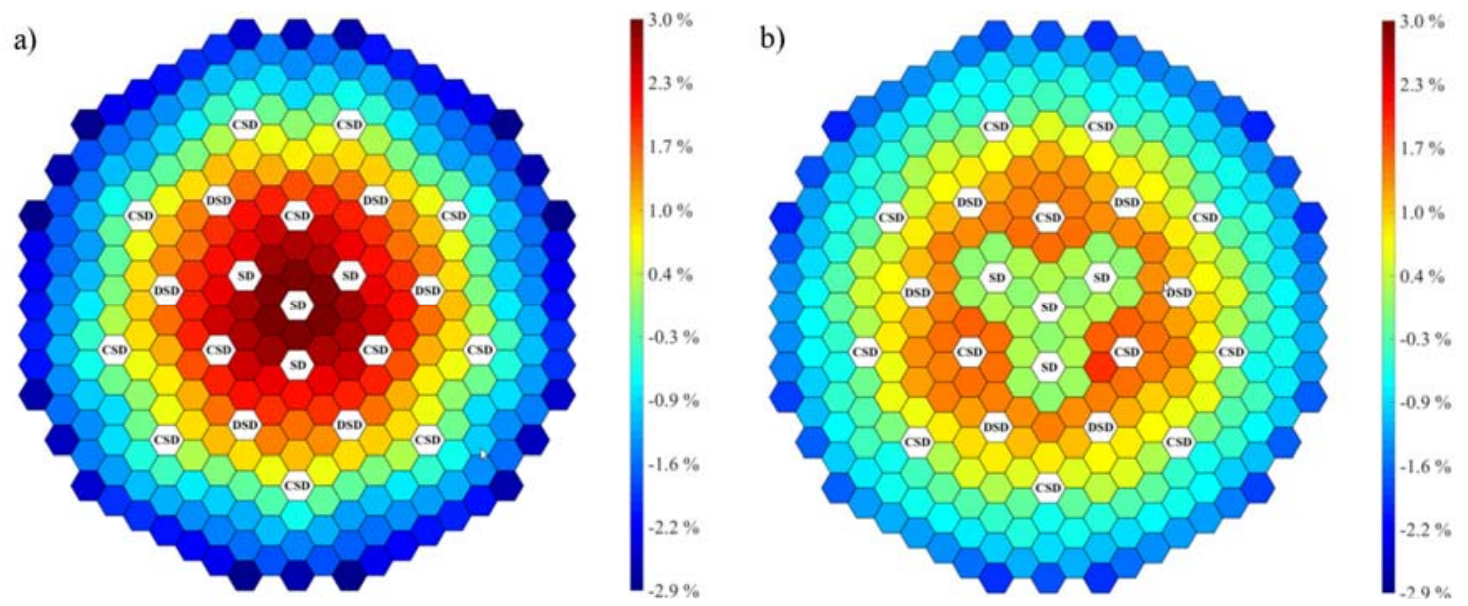

Figure 5. Un-rodded core power maps - a) Model \#1, b) Model \#6.

Consequently, the results indicate that application of Serpent 3D capabilities improves the k-eff, while the SPH factors play an important role in the alignment of the overall core reactivity and power map coherence in deterministic calculations. However, when improving the consistency of the overall results, very often a balance between the radial power map and k-eff accuracies has to be struck. 


\section{SUMMARY AND CONCLUSIONS}

The goal of this study was to test the feasibility of applying the Serpent-DYN3D sequence to the calculations of an SFR core of a non-standard design - ASTRID-like. The results were compared against the reference Serpent full-core simulations. Simultaneously, an attempt to qualitatively assess the approximate time effort spent on new XS generation or correction methods against the accuracy achieved was made.

In the case of accuracy, the application of more complex models and methods affects the reliability of the DYN3D simulations, as it, in general, improves the agreement in k-eff and radial power distribution. It has a limited impact on the axial power distribution. In general, replacing XS produced with 2D single assemblies (for fuel regions) by XS created with 3D single assemblies (for fissile and fertile regions in the inner core) or with 2D fuel-reflector models (for fertile fuel regions in the outermost fuel assemblies) is recommended. Moreover, correcting the XS generated with 2D super-cells with SPH factors, for nonmultiplying regions of the inner core, adjacent to the fuel, is highly advised. Optionally, XS corrected this way may be applied to these adjacent fuel regions inside the core. However, care should be taken when introducing XS generated or corrected with more complex methodologies, as their simultaneous application to various core regions may worsen the overall results. They may influence one another.

In the case of an attempt to assess the approximate efforts needed to prepare the calculations against the achieved accuracy, it should be noted that at a certain point the time efforts seem to outweigh benefits. In the case of Model \#4 to \#6, a full-core Serpent MC simulation can be programmed and processed with a similar time effort and more accurate results. However, when the XS are adequately prepared, and the reference calculations give results of acceptable accuracy, and multiple core configurations in the same conditions are to be tested, it may be stated with certainty that fewer resources are required to interchange the XS configurations and run DYN3D calculations (for example for rod insertion) than to prepare and run a new MC full-core simulation.

To sum up, Serpent-DYN3D sequence may be used for complex, heterogeneous SFR core designs deterministic calculations. However, there are diminishing returns to using the more complex XS generation methods. It is more profound if the complexity of the core's design requires reconstruction of significant regions of the core for XS generation. Therefore, applying XS generation methods as described in Model \#4 would be advisable, based on the quantitative analysis of the quality of the results and qualitative analysis of the effort required to obtain these results. Finally, it should also be noted that the enhancements in the results are clearly more visible in the case of the fully rodded state.

With regards to future work, it could be viable to provide a quantitative analysis of the efforts required to prepare increasingly complicated models that resemble a larger percentage of parts within the advanced reactors core for the XS generations. Moreover, further research on the applicability of 2D fuel-reflector models for modelling of outermost fuel assemblies could be undertaken. It would be advisable to asses why the coupling of the radial reflector and adjacent fuel sub-assemblies in space and energy provides the most realistic XS for the fertile fuel sub-assemblies (SA), and not for fissile fuel SA.

\section{REFERENCES}

1. E. Fridman and E. Shwageraus, "Modeling of SFR cores with Serpent-DYN3D codes sequence," Annals of Nuclear Energy, 53, pp. 354-363 (2013).

2. E. Nikitin, E. Fridman, and K. Mikityuk, "Solution of the OECD/NEA neutronic SFR benchmark with Serpent-DYN3D and Serpent-PARCS code systems," Annals of Nuclear Energy, 75, pp. 492497 (2015). 
3. E. Nikitin, E. Fridman, and K. Mikityuk, "On the use of the SPH method in nodal diffusion analyses of SFR cores," Annals of Nuclear Energy, 85, pp. 544-551 (2015).

4. E. Nikitin and E. Fridman, "Modeling of Phenix End-of-Life control rod withdrawal tests with the Serpent-DYN3D code system," IAEA-CN-245, Yekaterinburg, Russian Federation, June 26 - 29, 2017 (2017).

5. E. Nikitin and E. Fridman, "Extension of the reactor dynamics code DYN3D to SFR applications - Part I: Thermal expansion models," Annals of Nuclear Energy, 119, pp. 382-389 (2018).

6. E. Nikitin and E. Fridman, "Extension of the reactor dynamics code DYN3D to SFR applications - Part II: Validation against the Phenix EOL control rod withdrawal tests, "Annals of Nuclear Energy, 119, pp. 411-418 (2018).

7. E. Nikitin and E. Fridman, "Extension of the reactor dynamics code DYN3D to SFR applications - Part III: Validation against the initial phase of the Phenix EOL natural convection test, "Annals of Nuclear Energy, 119, pp. 390-395 (2018).

8. E. Nikitin and E. Fridman, "Modeling of the FFTF isothermal physics tests with the Serpent and DYN3D codes," Annals of Nuclear Energy, 132, pp. 679-685 (2019).

9. GIF, "Technology Roadmap Update for Generation IV Nuclear Energy Systems," https: //www.gen-4.org/gif/upload/docs/application/pdf/2014-03/gif-tru2014.pdf (2017).

10. CEA, 4th-Generation Sodium-cooled Fast Reactors - The ASTRID Technological Demonstrator, Technical Report, CAE, France (2012).

11. K. Sun, Analysis of Advanced Sodium-cooled Fast Reactor Core Designs with Improved Safety Characteristics, PhD Thesis, EPFL, Laussane, France (2012).

12. N. Oshkanov, O. Saraev, M. Bakanov, P. Govorov, O. Potapov, Y. M. Ashurko, V. Poplavskii, B. Vasil'ev, Y. L. Kamanin, and V. Ershov, "30 years of experience in operating the BN-600 SodiumCooled Fast Reactor," Atomic energy, 108(4), pp. 234-239 (2010).

13. H. Yamaki, K. Inoue, A. Suzuoki, and S. Shingai, "Current status of liquid metalcooled fast breeder reactor safety research," Hitachi Hyoron, 64(8), pp. 611-614 (1982).

14. A.Tassone, A.Smirnov, and G.Tikhomirov, "Specifications for a coupled neutronics thermalhydraulics SFR test case," Journal of Physics: Conference Series, 781(1), IOP Publishing (2017).

15. S. Bortot, F. Alvarez-Velarde, E.Fridman et al. "European benchmark on the ASTRID-like lowvoid-effect core characterization: neutronic parameters and safety coefficients," Proceedings of ICAPP 2015, Nice, France, Mary 03-05, 2015 (2015).

16. F. Gabrielli, A. Rineiski, B. Vezzoni et al., "ASTRID-like Fast Reactor Cores for Burning Plutonium and Minor Actinides," Energy Procedia, 71, pp. 130-139 (2015).

17. W. Rydlewicz, Modelling of the ASTRID-like Sodium-cooled Fast Reactor with the SerpentDYN3D code system, MPhil Thesis, University of Cambridge, Cambridge, UK (2017).

18. A. Kavenoky, The SPH homogenization method, Technical Report, CEA Centre d'Etudes Nucleaires de Cadarache, Cadarache, France (1978).

19. A. Hebert and A. Kavenoky, Development of the SPH homogenization method, Technical Report, CAE, Centre d'Etudes Nucleaires de Cadarache, Cadarache, France (1981).

20. A. Hebert, "A consistent technique for the pin-by-pin homogenization of a pressurized water reactor assembly," Nuclear Science and Engineering, 113(3), pp. 227-238 (1993).

21. T. Courau, M. Cometto, E. Girardi, and N. Schwartz, "Elements of validation of pin-by-pin calculations with the future EDF calculation scheme based on APOLLO2 and COCAGNE codes," Proceedings of the 2008 International Congress on Advances in Nuclear Power Plants-ICAPP'08, Anaheim (CA), USA, June 9-12, 2008, pp. p. 2439-2448 (2008).

22. U. Grundmann and S. Mittag, "Super-homogenisation factors in pinwise calculations by the reactor dynamics code DYN3D," Annals of Nuclear Energy, 38(10), pp. 2111-2119 (2011). 